\title{
LE MYTHE DE PAUL ET VIRGINIE À TRAVERS TROIS ROMANS FRANCOPHONES DE L'OCÉAN INDIEN
}

\begin{abstract}
[...] L'histoire des deux enfants se transforme en légende, ou plus exactement en "mythe», " une histoire que tout le monde connaît déjà », selon la définition minimale mais très parlante que le romancier Michel Tournier propose de cette notion ${ }^{1}$.
\end{abstract}

La popularité de Paul et Virginie, sorte d'exemplum paru dans le quatrième volume des Études de la nature, en 1788, s'est maintenue, tant bien que mal, jusqu'à la fin du XIX ${ }^{\mathrm{e}}$ siècle. Jean-Michel Racault, qui en a retracé plus d'une fois la fortune, a aussi cherché à en expliquer les aléas ${ }^{2}$. Atala de Chateaubriand (1801) et Indiana de George Sand (1832) offrent deux reprises les plus connues dans l'Hexagone, dont l'une se situe en Amérique et l'autre la plupart du temps en France, avec juste un épisode réunionnais, pourtant décisif. Jean-Michel Racault a été relayé par Valérie Magdelaine-Andrianjafitrimo ${ }^{3}$, qui a ajouté à sa liste

${ }^{1}$ J.-M. Racault, « Introduction » à Paul et Virginie (P\&V, p. 42 : édition de référence, sauf exception; voir la liste des textes sources à la fin de l'article). La phrase citée de M. Tournier vient du Vent Paraclet, Gallimard, collection « Folio », Paris 1981, p. 189.

${ }^{2} \mathrm{Par}$ ex. «Réception, réminiscences et réécritures » dans l'Introduction ci-dessus ( $\mathrm{P} \& \mathrm{~V}$, pp. 40-47).

${ }^{3}$ V. Magdelaine-Andrianjafitrimo, « Décalages, réinterprétations et sacrilèges. Les échos de Paul et Virginie dans quelques textes de la littérature contemporaine de l'océan Indien ", [dans :] Bernardin de Saint-Pierre et l'océan Indien, J.-M. Racault, Ch. Meure, A. Gigan (dir.), Classiques Garnier, Paris 2011, pp. 485-500. 
deux textes : Noëlla de Georges Azéma $(1864)^{4}$ et Une Île où séduire Virginie (2007) de Jean-François Samlong. Extension qui permet d'interroger les effets littéraires de la réappropriation que font du couple mythique les écrivains d'origine mauricienne et réunionnaise. Dans deux essais consacrés l'un à Loys Masson (dont Les Noces de la vanille. Roman, de 1962, nous intéresse ici) et l'autre à JeanMarie Gustave Le Clézio (son Chercheur d'or de 1985), Jean-Michel Racault analyse l'empreinte des hantises et ambitions qui se décèlent dans leurs textes, inscrits dans les grands débats historiques ou éthiques ${ }^{5}$.

Le logos — parole réfléchie — d'un auteur est pour ainsi dire mis au service d'un mythos réactualisé ou action d'un ou de plusieurs personnages qui incarnent un conflit essentiel à la communauté - laquelle le revit par leur intermédiaire ainsi que la solution de ce conflit ${ }^{6}$. Jean-Michel Racault y voit la force d'attraction que représente l'histoire de Paul et Virginie pour leurs contemporains, voire pour les premiers romantiques en Europe, alors que dans l'île Maurice leur gloire va grandissant jusqu' au $\mathrm{XX}^{\mathrm{e}}$ siècle, les tendres « enfants de la nature » étant devenus des « ancêtres glorieux » pour les mauriciens :

Quant aux fonctions que peut assumer le mythe de Paul et Virginie [...] le roman apparait surtout comme une variante du mythe de l'Éden tel qu'il est rapporté dans la Genèse, voire comme une transposition du scénario chrétien innocence/chute/châtiment/rédemption : l'état paradisiaque initial, brisé par l'émergence de la tentation sensuelle, est retrouvé et éternisé dans cet au-delà lumineux auquel la mort donne accès, en même temps que redeviennent compatibles l'amour et l'innocence. $(\mathrm{P} \& \mathrm{~V}, \mathrm{p} .47)^{7}$

Voilà une signification « générale », perçue à travers des siècles et des publics divers, à laquelle se superposent des significations particulières, adoptées pour cette pastorale convertie en roman ${ }^{8}$ pendant les deux cents ans écoulés depuis sa

${ }^{4}$ Repris en feuilleton dans Le Moniteur de La Réunion, ${ }^{\text {os }} 54$ à 72 , du 6 juillet au 7 septembre 1878 (Bernardin de Saint-Pierre ..., p. 486, note 2).

${ }^{5}$ M. Racault, "L'œuvre "indianocéanique" de Loys Masson : une poétique métisse » et «L'Écriture des pierres : Fiction généalogique et mémoire insulaire dans Le Chercheur d'or et Voyage à Rodrigues de Le Clézio », [dans :] idem, Mémoires du Grand Océan. Des relations de voyages aux littératures francophones de l'océan Indien, Presses de l'Université Paris-Sorbonne, collection « Lettres Francophones », Paris 2007, pp. 203-242.

${ }^{6}$ Définition du mythe d'après A. Dabezies (« Miroirs du mythe », Revue Europe : Faust, $\mathrm{n}^{\text {os }}$ 813-814, 1997, p. 4), cité par M. Klik, Teorie mitu. Wspótczesne literaturoznawstwo francuskie (1969-2010), Wydawnictwa Uniwersytetu Warszawskiego, Warszawa 2016, p. 153. Voir aussi W. Stróżewski, «Logos i mythos », [dans :] Człowiek wobec świata na przełomie wieków. Nowe i dawne wzorce duchowości, M. Kudelska (dir.), Collegium Columbinum, Kraków 2001, pp. 17-36.

7 J.-J. Rousseau, maître à penser de Bernardin de Saint-Pierre, trouva une recette analogue pour « concilier l'amour et l'innocence ", dans La Nouvelle Hélö̈se : l'innocence de Julie et de Saint-Preux, recouvrée par leur « rédemption » sentimentale aux côtés de l'athée Wolmar, serait le mieux garantie par la mort (apparemment accidentelle) de l'héroïne, ce qu'elle-même finit par avouer dans sa dernière lettre à l'ancien amant (J.-J. Rousseau, Julie ou la Nouvelle Héloïse. [...], R. Pomeau (dir.), Classiques Garnier, Paris 1960, p. 728).

${ }^{8}$ J.-M. Racault, « Genres : pastorale ou roman ? », [dans :] « Introduction » (P\&V, pp. 16-19). 
publication. Mis au service de «la quête d'une légende fondatrice d'une communauté nationale $»^{9}$, la mauricienne - notamment après la conquête anglaise de l'île en 1810 - , le mythe des " gémeaux » créoles a depuis reculé devant les accusations d'eurocentrisme. L'évolution, au sens de dégradation, que le mythe a vécue à l'époque postcoloniale, invite le chercheur d'aujourd'hui, comme le public d'étudiants, à scruter ce qui, dans la parole de l'écrivain et le message véhiculé par son livre, serait anachronique. Paradoxalement, moins que les contenus pérennes, comme l'ambiguïté de la nature exotique exaltée, ce sont ceux les plus contingents qui peuvent intéresser, telles les relations de domination ou le motif du sacrifice, dont l'enjeu reste brûlant d'actualité pour nous.

Trois romans, alors, qui reprennent le mythe bernardinien dans l'espace de cent vingt ans ; la réconciliation ou le dépassement des intérêts opposés dans une réalité immédiate paraissent impossibles à leurs auteurs. Sans le recours à un niveau qui transcende l'espace-temps de l'histoire - la force des sentiments ou un au-delà réconfortant - le conflit y demeure irréductible. Or, les romanciers cherchent à pallier cette insuffisance : telle parole, simple mot ou métaphore, sinon tel personnage ou tel objet, porteur d'un sens (logos) spécifique, suppléent à l'absence d'une solution explicite et inconditionnelle au niveau de l'histoire ( $m y$ thos). Colonial ou postcolonial, le mythe ancien sous des habits nouveaux nous invite à entrer dans un dialogue entre siècles et cultures.

\section{NOËLLA, LE MYTHE RENDU INUTILE}

Une certaine solution est encore possible aux yeux du narrateur de Noëlla, et qu'à ses yeux, il faut souligner. Mais elle dépasse l'emploi réservé au mythe en question. Le lecteur qui parcourt l'histoire de la malheureuse princesse de Wolfentbuttel, morte des suites de blessures reçues de son époux, le tzarevitch Alexeï Petrovitch Romanov ${ }^{10}$, mais ressuscitée par la grâce de la fiction, risque un double malaise : sevré de l'histoire du couple adolescent, il doit affronter les avis du narrateur, omniscient et anonyme, concentré sur la mère de l'hérö̈ne. Par ces derniers, il se voit même obligé de repenser la visée du roman ${ }^{11}$. Madame

\footnotetext{
${ }^{9}$ Ibidem (P\&V, p. 47).
}

${ }^{10}$ Dans l'histoire vraie, il s'agit de Charlotte Christine Sophie de Brünswick-Lüneburg (16941715), la belle-sœur de l'empereur Charles VI, qui fut maltraitée après la naissance du futur tzar Pierre II Romanov ; comme sa sœur Nathalie, ce dernier mourra sans héritier.

${ }^{11}$ Le récit fait allusion à la fuite incognito de la jeune princesse de Saint-Pétersbourg où elle aurait craint pour la vie de l'enfant qu'elle portait dans son sein (N, p. 75). Le narrateur renchérit, à propos de la fin de la prétendue Madame Dauban, morte peu après Noëlla : "C'était une chose digne de pitié de voir cette femme, à qui n'auraient pas manqué les consolations d'une illustre et nombreuse famille, mourant dans une misérable cabane, n'ayant que deux pauvres esclaves pour la plaindre. Triste effet des unions mal assorties! Graves enseignements pour celles qui ne savent point trouver dans la résignation des compensations à leurs souffrances, comme si le mariage n'était pas 
Dauban, avatar de Mme de La Tour, mère de Virginie — la jeune fille est le modèle implicite de Noëlla - finit par éclipser sa fille. Car ce que devient la mère après la mort de Noëlla décide du message du roman. D'emblée, le lecteur avise qu'il aurait affaire à un roman de conversion et de repentir : la conversion au catholicisme dans le cas de Noëlla, élevée en luthérienne par sa mère (N, pp. 41-44), est suivie de loin par celle de Mme Dauban, qui devient catholique sur l'insistance de sa fille mourante ( $\mathrm{N}$, pp. 126-127). Le repentir de la mère en deuil, qui dans son chagrin allait jusqu'à blasphémer (N, pp. 130-131, 136-137), donne un sens à l'irruption finale de Raoul devenu prêtre. Cet avatar de Paul (surtout pour la consonance) se manifeste devant Mme Dauban à l'agonie : " Lorsqu'elle eut rendu les derniers soupirs et qu'il eut prononcé les dernières paroles, l'ecclésiastique se retira ne voyant qu'une âme à sauver dans ce lieu, témoin de jeux de son enfance et des premières émotions de sa jeunesse, et n'ayant conservé de tous ses souvenirs que les huit jours qu'il avait passés sur le piton des Salazes ${ }^{12} »(\mathrm{~N}$, p. 137). Le bref retour dans ce « lieu de mémoire» sentimental le confirme dans sa vocation.

Paradoxalement, cet orphelin recueilli par le Père Étienne (évangélisateur efficace de la paroisse de Mme Dauban), dont les parents (la mère de sang mêlé) auraient péri à Madagascar, semble mieux parrainé grâce à ce religieux modèle que ne le sont Noëlla ou Mme Dauban. Car le Père Étienne, ayant pris une décision peu vraisemblable chez ce saint homme, laisse la mère inconsolable dans son chagrin : " Il retourna dans son pays pour y consacrer au repos le peu qui lui restait d'une vie pleine de charité, de dévouement et de vertu » (N, p. 130). Si l'on voulait chercher des parallélismes entre l'histoire des enfants du roman de Bernardin et de celui d'Azéma, la première surprise est la profonde dissymétrie, annonciatrice de rupture, dans la vie des deux couples.

Chez Bernardin, les deux mères partagent entre elles et leur voisin narrateur le « fatal secret » qui leur a valu la rupture d'avec leurs familles ; Mme de La Tour réussit même à imposer à Virginie le devoir de renouer avec son milieu d'origine ; elle en est bien punie, puisqu'elle suit la dernière sa fille au tombeau, après les autres membres de la « petite société ». Mme Dauban garde pour elle le secret de sa naissance, alors que, avertie à temps, Noëlla aurait pu dissuader sa mère de compter sur son retour en Europe et Raoul n'aurait pas été écarté, ce qui a décidé de la douloureuse rupture entre lui et Noëlla. Par sa «faute » d'épouse fuyant ses devoirs, Mme Dauban rend nécessaire sa repentance, à laquelle la mort de Noëlla et le retour de Raoul en prêtre donnent l'occasion. Les deux événements deviennent deux épisodes subalternes, motivant ce qui ne paraissait qu'une toile

pour elles une vie de sacrifices, d'amour, de peines et de dévouement. Il semblait que la mort de sa fille n'eût pas suffi pour expier l'oubli qu'elle avait fait de ses devoirs d'épouse. [...]» (N, p. 133).

${ }^{12}$ Massif montagneux au centre nord-est de l'île, dans lequel Raoul s'est retiré dès qu'il avait compris que Mme Dauban ne lui donnerait pas sa fille en mariage ; dans ce désert, il a cherché à éprouver sa décision de devenir prêtre. 
de fond déterminante pour l'histoire d'amour, si la structure du modèle bernardinien avait été observée.

Plus désintéressée et plus authentique, Virginie déploie ses vertus devant Paul et la famille, aux yeux des voisins émerveillés; elle ne se dément pas en Europe. Noëlla se lasse d'être vertueuse dès qu'elle perd l'espoir de voir Raoul revenir vers elle ; résignée, elle se laisse alors mourir. Une issue sentimentale, donc, alors que ce sont les éléments déchaînés de l'océan qui ont occasionné la fin de Virginie vouée à un sort tragique.

Paul a été le compagnon de Virginie depuis sa naissance ; Noëlla ne fait la connaissance de Raoul qu'âgée de dix ans environ (avant sa première communion) ; avant, c'est à sa mère qu'elle offre ce que Paul a offert à Virginie (des fruits, un nid d'oiseau [N, p. $31 ; \mathrm{P} \& \mathrm{~V}$, p. 128]). Ses souffrances dues à la séparation rappellent aussi celles de Paul (N, pp. 97, 121-127 ; P\&V, pp. 248, 262). La mère occupe la position centrale de Virginie. Sa substitution à l'héroïne éponyme se confirme encore dans le contexte biblique, dans l'actualisation de l'histoire de Ruth et Booz. Si Mme de La Tour faisait la lecture de la Bible aux enfants, inspirant à Virginie des pantomimes où avec Paul ils préfiguraient leur mariage $(\mathrm{P} \& \mathrm{~V}$, pp. 164-165), c'est Noëlla qui lit le Livre de Ruth, entre autres, à sa mère. Il est alors clair que les incarnations de Ruth et Booz, ce seraient Mme Dauban et son voisin Ducheman. Une identification impossible, d'ailleurs, car pour la princesse allemande devenue veuve, même incognito il ne saurait être question d'un pareil remariage (N, pp. 32-33).

La réduction de la place réservée à Noëlla dans le roman d'Azéma et la « déchéance » de cette héroïne par rapport à son prototype bernardinien sont encore sensibles si l'on rapproche leurs devises de bienfaisance. Selon Virginie, « on ne fait son bonheur qu'en s'occupant de celui des autres » (P\&V, p. 168) ; Noëlla soutient : «Tant que les autres ne manquent de rien, nous en aurons toujours assez » (N, p. 118). Si le mot clé dans la bouche de Virginie est le bonheur qu'il s'agit de faire, Noëlla met l'accent sur l'opposition entre manquer et avoir, bien « bourgeoise » dans ses motivations, comme elle l'avoue elle-même (N, p. 114).

Virginie avait une vertu plus " holistique », elle invitait de pauvres filles pour faire plaisir à sa mère, et si elle leur donnait ses habits, ce n'était qu'avec le consentement de celle-ci (P\&V, pp. 167-168). Paul était toujours là, admiratif. Noëlla se laisse distraire dans son chagrin, après le départ de Raoul, en regardant danser ou travailler les autres, sinon en leur portant de l'aide. Elle se fait gronder par sa mère qui trouve que la fille donne trop d'habits aux pauvres $(\mathrm{N}, \mathrm{pp}$. 117118). À qui la faute ?, pourrait demander le lecteur.

L'enterrement de Virginie devient une fête « interculturelle », où se manifeste le syncrétisme religieux bien connu aujourd'hui à la Réunion (P\&V, pp. 246-248); l'enterrement de Noëlla a lieu en l'absence de sa mère : était-elle malade? Évanouie ? Elle demande à sa servante comment s'est passée la cérémonie (N, p. 132). 
Ce roman religieux, qui fait l'éloge de la vocation sacerdotale (N, pp. 82-83) et de l'efficacité de la prière (N, pp. 136-137), sacrifie assez vite le bonheur d'une jeune, belle et bonne personne pour en faire un outil de conversion pour son ami et pour sa mère. Finalement, c'est moins l'au-delà des bienheureux, entrevu dans les songes convergents de Marguerite et de Mme de La Tour (P\&V, pp. 260-261), qui transparaît dans le dernier paragraphe, que plutôt un impératif d'humilité prescrit aux humains ici-bas. Les survivants ou porteurs du message seront les deux esclaves à qui Mme Dauban, avant de mourir, avait déclaré rendre la liberté avec un legs : " J'ai perdu tous mes amis, ô mes chers noirs! Vous seuls me restez. Après ma mort, vous serez libres ; partagez-vous cette habitation, vivez heureux et pensez à ma fille » (N, p. 132).

Selon Valérie Magdelaine-Andrianjafitrimo, " l'épuisement progressif de l'influence de l'œuvre ", c'est-à-dire de la force du mythe de Paul et Virginie, permet « de nous interroger sur le rapport de filiation et de rupture ambigu que la littérature indianocéanique entretient avec la France et son canon littéraire $»^{13}$. Azéma, connu pour son Histoire de l'île Bourbon (1859) ${ }^{14}$, achève la « créolisation » du mythe, potentialisée déjà chez Bernardin : c'est le point de vue insulaire qui l'emporte comme critère de conduite modèle ${ }^{15}$. À sa mère, qui souhaite l'établir en Europe, selon le haut rang réservé à sa famille, Noëlla, «pauvre créole, qui ne conna[ît] que les travaux de la campagne », répond : « Ne sacrifions pas à des idées de grandeur le repos dont nous jouissons » (N, p. 115). Elle souligne le contraste entre « ces cours, où tout n'est que déguisement, mensonge et perfidie; où les cœurs sont masqués comme les visages; où l'étiquette est tout; où le mérite n'est rien, s'il n'est rehaussé par l'éclat du nom; où les vertus sont trop bourgeoises pour y avoir accès », et la réalité coloniale idyllique : « Ici, chacun jouit d'une douce égalité, chacun est bienveillant, hospitalier, miséricordieux ; nul ne porte envie à son voisin; tous se disputent le plaisir d'obliger celui qui est dans le besoin. Où trouver des cœurs meilleurs, des mariages mieux assortis?» (N, p. 114). Noëlla se fait ainsi porte-parole de la tradition qui idéalise les rapports sociaux au début de la colonisation de l'île Bourbon-la Réunion, et qui l'oppose même, sur ce point, à son « île sœur », Maurice ${ }^{16}$. Elle enseigne sa mère comme le fait le voisin expérimenté vis-à-vis de Paul (P\&V, pp. 216-227) : par son refus

${ }^{13}$ V. Magdelaine-Andrianjafitrimo, op. cit., p. 485.

${ }^{14}$ Sur la page de titre de Noëlla, G. Azéma est présenté comme l'auteur de cette monographie et le roman comprend des pages d'histoire hautes en couleur (la fin de la colonie de Fort-Dauphin, en 1674, racontée par le grand-père de Raoul et rapportée par le P. Étienne à Mme Dauban). L'île de Bourbon est le premier nom français de la Réunion, appelée auparavant Mascareigne.

${ }^{15}$ Le premier sens du mot créole est « habitant des îles, colon » (éthymologie : esp. criollo, le coq).

${ }^{16}$ Un reflet de ces origines égalitaristes transparaît dans la dénomination de créoles réservée, de nos jours encore, à tous les habitants de la Réunion, contrairement à l'île Maurice, où le cloisonnement ethnique, social et économique a toujours été beaucoup plus prononcé et où ne sont appelés créoles que les Afro-mauriciens de confession chrétienne (pour la plupart catholiques romains). 
de partir et par son jugement de valeur puissant sur le monde insulaire opposé à l'Europe, fondement de la conscience identitaire créole, elle aurait pu aider à construire l'identité réunionnaise :

[...] À l'inverse de sa mère, européenne, qui privilégie la filiation, Noëlla a choisi de devenir fille de sa terre d'élection. À l'économie de l'héritage et de l'argent transmis indûment avec le titre s'oppose une économie du travail de la terre et de sa fructification. Le texte propose un modèle idéologique apte à servir la classe de planteurs, toujours en délicatesse avec leurs origines. Les vertus créoles, idéalisées par Bernardin de Saint-Pierre, se veulent ici réhistoricisées ${ }^{17}$.

Son enracinement, productif d'un mythe fondateur réunionnais, à l'instar de celui que Paul et Virginie ont fourni aux Mauriciens, n'a pourtant pas trouvé d'écho ${ }^{18}$. Un détour par la gloire littéraire parisienne était-il nécessaire ? Ni l'un ni l'autre n'ont joué en faveur du roman d'Azéma. Déphasé ? Le public parisien était alors gagné au scientisme, raffinant sur l'école du Parnasse fondée par Leconte de Lisle, un autre fils de Bourbon-la Réunion, et se préparant à goûter au naturalisme ; il devait être peu motivé à recevoir une églogue chrétienne. Son message se recommandait plutôt aux colons dont le roman cherchait à magnifier l'esprit humble et charitable.

\section{LES NOCES DE LA VANILLE, MYTHE IMPUISSANT ET SOUILLÉ}

Contrairement à Georges Azéma, notable réunionnais qui publie à Paris mais vit dans le Sud, Loys Masson, né en 1915 dans une famille franco-mauricienne et arrivé en France, demeurée son pôle culturel, à la veille de la Seconde Guerre mondiale, y vivra et mourra trente ans après. Il ne parviendra pas à une reconnaissance nationale. Loys Masson a pourtant fréquenté les élites littéraires de son temps, depuis Louis Aragon jusqu'à Claude Roy, les communistes et les catholiques, ayant tissé des liens avec les uns (pour un court temps) comme avec les autres (plus durables), avant de sombrer dans l'alcoolisme qui a abrégé ses jours. De ses neuf romans, Le Notaire des noirs (1961) a été réédité à la fin du XX siècle, dans une anthologie de proses françaises de l'océan Indien ${ }^{19}$. Les Noces de la vanille, dont il sera question ici, ont connu une seule réédition ${ }^{20}$.

Mieux connu pourtant que Noëlla, le roman de Masson se résumerait, dans l'analyse de Jean-Michel Racault, à une " pastorale perverse » dans laquelle le Bien et le Mal auraient interverti leurs rôles ${ }^{21}$ : un intendant diabolique au visage

17 V. Magdelaine-Andrianjafitrimo, op. cit., p. 491.
18 Ibidem.
19 Océan Indien. Madagascar, Réunion, Maurice, S. Meitinger, J.-C. Carpanin Marimoutou (dir.), Omnibus, Paris 1998.

20 Éditions de l'Océan Indien, Île Maurice, 1981.

21 J.-M. Racault, «Paul et Virginie, version infernale : la pastorale perverse des Noces de la vanille (1962) », [dans :] Mémoires du Grand Océan..., p. 217. 
difforme (Esparon) cherche à se faire ami d'un couple d'adolescents, le narrateur anonyme et sa petite amie créole Marie-Thérèse. Il observe leurs amours naissantes et vite corrompues par une sensualité débordante. Cet anti-Quasimodo les compare à " de la vanille qui marche », image qui prolonge son extase par substitution de l'amour interdit : ce célibataire, vierge et alcoolique (saisonnier), s'emploie à féconder les fleurs de la vanille, la plante étant incapable d'y procéder toute seule. Le cadre est une nature elle-même malade d'abondance de vie qui la fait tourner à la mort. La féminité à peine éclose de la jeune fille lui attire, avec l'entremise d'Esparon, une fin fatale : elle mourra d'hemmorragie en couches, ayant mis au monde l'enfant de l'homme qui avait promis de veiller sur la jeune femme jusqu'au retour de son fiancé. De retour, adulte, ce dernier découvre la trahison tout en apprenant la mort de la jeune femme. Esparon sombre dans la folie, mais le narrrateur croit le retrouver en la personne de son charismatique chef de maquis nouveau-calédonien, le destinataire impossible du récit autobiographique du jeune homme. Sa conclusion s'en ressent : "Chacun naît en puissance d'un paradis terrestre. Chacun le perd. Mais Satan qui me l'a fait perdre jadis est aujourd'hui devenu archange par vos soins. Que vous ai-je donc fait, mon Dieu ? Qu'avais-je fait à ce visage-là ?» (NV, p. 166). Dieu même, figure paternelle, en souffre, devenu un être par excellence ambigu ${ }^{22}$.

Selon Racault, à l'origine, il y aurait « la crise de l'image paternelle» ou une « donnée invariante de l'œuvre de Masson, et plus généralement - il conviendrait de se demander pourquoi - de l'essentiel de la production romanesque 'indianocéanique' $[\ldots] »^{23}$.

En creux, le roman démontre une vérité bien connue des psychologues : l'absence d'affection parentale prive l'enfant de repères dans la vie. C'est à travers l'amour des parents, motivation de leurs actes, dont les actes de parole, que l'enfant apprend à distinguer le bien du mal, donc à se tenir à distance du mal, à défaut de pouvoir le dominer, tout en recherchant le bien. Telle est du moins l'optique chrétienne avec laquelle le romancier malgré tout reste en phase, à en croire son ami Claude Roy ${ }^{24}$.

Paul et Virginie, Noëlla et Raoul ont eu beau partir dans la vie par des sentiers différents, à l'origine, chacun d'eux avait reçu, qui de sa mère, qui de son grand-père ou voisin, voire d'un ecclésiastique, un capital affectif et moral qui les

${ }^{22}$ Chez Samlong, le vieillard ami du jeune couple se trouve aussi défiguré (V. Magdelaine-Andrianjafitrimo, op. cit., p. 491).

23 J.-M. Racault, Mémoires du Grand Océan..., p. 224.

24 «[...] Ce sont peut-être les plus étranges prières qu'un moderne ait jamais exhalées si on excepte les noires prières des incroyants religieux, d'Artaud à Georges Bataille », constate Claude Roy, à propos du recueil de Masson, Pour la Passion Jésus-Christ douze poèmes d'un indigne (1966). Voir C. Roy, «Esquisse d'un portrait de Loys Masson par... », [dans :] L. Masson, Les Tortues, chez Robert Laffont, collection « Bibliothèque romanesque », Paris 1970, p. XVII. 
aiderait à supporter les épreuves. Cela n'a pas été le cas du narrateur des Noces de la vanille.

Dès le second chapitre, nous apprenons que cet enfant solitaire, asthmatique et vulnérable demeure l'otage des échecs professionnels et des frasques sentimentales de son père. Vaines promesses ou menaces, voire éclipses ou malentendus, voilà par quoi ses parents répondent à son affection, celle d'un être faible qui cherche de l'appui contre un monde étranger, voire hostile. Au début des années trente du vingtième siècle, obligée de quitter Madagascar, la famille s'établit à « la Morelle », une plantation de la Réunion en train de péricliter ; un cousin du père espère la remettre sur pied.

Au chagrin de la mère, surnommée madame Jeune-Fille par ses voisines dans son immaturité, elle n'aurait d'égal que son époux ${ }^{25}$ — se joint bientôt la nostalgie de l'enfant qui, à onze ans, doit laisser tout ce qui lui est cher, y compris le chat, pour s'embarquer vers l'inconnu. Dans cette situation encore, aucun appui sentimental :

Je pousse tout seul, comme je peux. Je sens bien que quelque chose me manque : une tendresse active, protectrice, peut-être même une surveillance. Je l'avais trouvée jusqu'à huit ans, j'avais du moins trouvé une confidente qui ne fût pas trop distraite dans Nénène Lucia, la vieille sakhalave ${ }^{26}$ qui avait pris soin de moi depuis ma naissance. [...] Mais l'argent devenait rare, il nous avait fallu la renvoyer... Quant à mon père, je ne l'ai jamais réellement intéressé. Il ne m'a pas caché en plusieurs occasions que, s'il m'aimait bien, j'avais cependant gâché sa vie en lui imposant des responsabilités qu'il n'avait pas désirées. [...] C'est un étranger que j'ai devant moi. (NV, pp. 15-16)

Sans secours, livré aux caprices d'Esparon, un éternel amoureux frustré, le narrateur se voit obligé d'éliminer le rival officieux. Son coup manqué, avec un revolver volé au père, lui vaudra une double punition. Aucun autre moyen pour lui de se défaire de l'intendant démoniaque, témoin de ses "péchés solitaires ", source d'angoisse initiale. L'égocentrisme du père et l'absence sentimentale de la mère aggravent la crise ${ }^{27}$. Le narrateur des Noces de la vanille, éternel coupable, semble plus victime de l'immaturité de ses parents et d'autres adultes que d'un augustinisme implacable dominant une terre trop riche en tentations. Même sa voix se perd, ne trouvant aucun interlocuteur, alors que son récit intérieur devait s'adresser à Manolo, chef d'insurgés calédoniens dont il a voulu, pour sa ressemblance avec Esparon, abréger les jours.

25 «Ils étaient faits pour être père et mère comme moi pour être pape... », selon des on-dit, cités par le narrateur (NV, p. 15).

${ }^{26}$ Les Sakalaves : l'une des ethnies les plus nombreuses de Madagascar vivant sur la côte occidentale de l'île. Connue pour ses légendes et rites, comme le bain des reliques royales.

${ }^{27}$ Le garçon demeure séparé de son amie, dans un univers sans charité, même de la part de sa mère. Il a beau rêver d'une "mère parallèle ", attentive, consolatrice, compréhensive : ce n'est pourtant pas que la sienne manque de sensibilité, mais elle manque de courage et de constance (NV, pp. 138-139). Plus tard, la même indolence chez les Payet, parents de Marie-Thérèse ; ils n'empêcheront pas Esparon de s'approcher de leur fille, pourtant « promise» au narrateur (NV, p. 166). 


\section{LE CHERCHEUR D'OR, OU LE MYTHE PRÉSERVÉ}

Pour voir combien ces qualités personnelles sont déterminantes, il suffit de faire la comparaison avec un autre couple d'enfants, Alexis et Laure, frère et sœur biologiques protagonistes du Chercheur d'or. Bénéficiaires de l'affection de leurs parents, qui s'aiment l'un l'autre aussi, ils ne perdent jamais le cap, même après une double catastrophe, le cyclone entraînant la faillite économique du père et de ses projets sociaux et scientifiques. Il a beau être perdant sur le plan matériel, pour ses enfants, qu'il a initiés à ses rêves et connaissances, il sera toujours un guide et ami, premier capitaine du navire Argo croisant dans le ciel (CDO, pp. 49-52, 61-64). Dans cette fiction biographique ${ }^{28}$, Jean-Marie Gustave Le Clézio transcrit l'aventure de son grand-père paternel Léon Le Clézio, lui prêtant le nom du grand-père maternel, Alexis L'Étang. Il opère ainsi la synthèse de deux traditions familiales enracinées dans l'île Maurice, île que l'écrivain a connue déjà adulte. La partie concernant la recherche du trésor du corsaire transporte le lecteur dans la troisième des îles Mascareignes, l'île Rodrigues ${ }^{29}$.

L'île Maurice, appelée île de France au temps de Bernardin, existe dans la mémoire du narrateur auto-diégétique par deux voix : celle de la mer, dont l'appel ouvre le roman $(\mathrm{CDO}, \mathrm{p} .11)$ et à laquelle le narrateur finit par céder pour retrouver la liberté, condition du bonheur (CDO, p. 375). La seconde est la voix de la mère, appelée à l'anglaise Mam, dont la mort précipite sa décision de rejoindre le large (CDO, pp. 24 et 374). D'autant qu'aucune des femmes qu'il a aimées ne peut le fixer auprès d'elle : ni sa sœur Laure, admiratrice du roman de Bernardin et qui finira pensionnaire d'un couvent à Port-Louis (CDO, pp. 360-361); ni Ouma, sa maîtresse, fille d'une Indienne et d'un manaf ou « Rodriguais des hauts » (descendant des noirs marrons). Celle-ci, éduquée par des bonnes sœurs en France, vit dans la montagne de Rodrigues avec son demi-frère Sri. D'ailleurs, Ouma disparaît contre son gré, déportée avec des ouvriers de plantation en grève, traités en réfugiés, embarqués pour être ramenés dans leur patrie - qui en Inde, qui au Pakistan, qui à Madagascar (CDO, pp. 369-370). À Mananava, près du camp de réfugiés, lieu marqué de souvenirs d'enfance, le narrateur croit revoir Ouma pour passer avec elle quelques jours à la sauvage, comme autrefois à Rodrigues (CDO, pp. 238-274; 363-367). Encore son bonheur éphémère semble-t-il plutôt un rêve éveillé que la réalité (CDO, pp. 352-353).

${ }^{28}$ Fiction autobiographique aussi, si l'on pense à la suite, Voyage à Rodrigues : journal (Gallimard, collection « Folio », Paris 1986), dont le narrateur s'identifie avec son grand-père.

${ }^{29}$ Un commentaire à ce roman se trouve dans un essai conçu comme journal du retour sur les lieux de mémoire familiaux, Voyage à Rodrigues, déjà cité. Deux romans postérieurs renouent encore avec le passé mauricien de la famille Le Clézio : La Quarantaine (1995) et Révolutions (2003). Voir L'Afrique et les Mascareignes de Jean-Marie Gustave Le Clézio, R.M. Atéba et K.R. Issur (dir.), Revue Mosaïques, hors-série ${ }^{\circ}{ }^{1}$, Éditions des Archives Contemporaines, Paris/Université de Maroua, août 2013. 
Après la banqueroute du père et la mort de la mère — deux coups qui arrivent à une vingtaine d'années de distance — Laure, l'aînée du narrateur, n'a pas les moyens d'offrir à son frère un refuge. Telle Virginie, inséparable de son frère durant leur enfance dans l'Enfoncement du Boucan, Laure a changé pendant l'absence d'Alexis, devenu homme du large, ensuite chercheur de l'or des pirates de Rodrigues, enfin soldat dans les tranchées de la Grande Guerre. Au bout de trente ans, le narrateur découvre en Laure une autre personne, d'ailleurs d'une grandeur d'âme incontestable, qui rappelle la dimension charitable de l'héroïne bernardinienne :

Je comprends tout d'un coup que, au cours de ces années d'exil, je l'ai perdue. Elle a suivi un autre chemin, elle est devenue quelqu'un d'autre, nos vies ne peuvent plus coïncider. Sa vie est parmi les religieuses de la Visitation, là où errent les hommes sans argent, sans foyer. Sa vie est auprès des Indiennes hydropiques, des cancéreuses, qui mendient quelques roupies, un sourire, des paroles de consolation. Parmi les enfants fiévreux au gros ventre, pour qui elle fait cuire des marmites de riz, pour qui elle va arracher un peu d'argent auprès des 'bourzois' de sa caste. [...] Plus tard, je la regarde tandis qu'elle prépare sa petite valise pour aller vivre chez les religieuses de Lorette. Son visage est redevenu calme, indifférent. Seuls ses yeux brillent, d'une sorte de colère. Elle entoure ses beaux cheveux noirs d'un châle de Mam, et elle s'en va, sans se retourner, avec sa petite valise de carton et son grand parapluie, haute et droite, et désormais plus rien ne peut la retenir ni changer sa route. (CDO, pp. 360-361)

Cette Virginie ne s'en va pourtant pas très loin - la misère la retient toujours prisonnière dans l'île - même si elle a l'air de disparaître à jamais de la vie du narrateur. Sa transformation avait déjà été pressentie pendant la lecture de son unique lettre, reçue par Alexis avant la Première Guerre, encore à Rodrigues, où Laure l' " appelle au secours ", contre son habitude de ne jamais se plaindre (CDO, pp. 248-249). Aux retrouvailles sur le quai (CDO, p. 309), il n'a reconnu Laure qu'à sa robe blanche, qu'elle avait portée au départ du frère pour Rodrigues, une vingtaine d'années auparavant. La robe blanche de Laure ${ }^{30}$ - en opposition au rouge, couleur associée à la métisse Marie-Thérèse des Noces de la vanille indique aussi sa chasteté virginale : déjà jeune fille, elle avait voulu aller à l'école " car elle voulait apprendre à travailler pour ne pas avoir besoin de se marier » $(\mathrm{CDO}, \mathrm{p} .104)$. Laure la porte à nouveau lors de leur escapade à vélo jusqu'à l'endroit qui fut le paradis de leur enfance. Frère et sœur contemplent du haut du col des Trois Mamelles les terres brûlées entre le Boucan et la Rivière Noire, la maison ayant été rasée par les nouveaux propriétaires (CDO, pp. 311-313) :

[...] il me semble qu'à force de regarder, avec cette lumière dorée qui illumine le rivage et la mer, je vais deviner les ombres des enfants que nous étions, en train de courir à travers les hautes herbes, pieds nus, visages griffés, habits déchirés, dans ce monde sans limites, guettant dans le crépuscule le vol des deux pailles-en-queue au-dessus du mystère de Mananava. (CDO, p. 313)

Le paysage d'enfance, la nature sont encore ici, dans leur beauté vierge, solidaires avec le mythe, comme la vallée des Prêtres entre Port-Louis et Pamplemousses chez

${ }^{30}$ Dans cette même robe, elle rappelle à Alexis la scène où Virginie « s'abrite sous une large feuille » de la pluie (CDO, p. 316). 
Bernardin de Saint-Pierre, alors que les Salazes chez Georges Azéma se voyaient confié un autre secret : celui de la naissance de la vocation sacerdotale chez Raoul.

Un élément encore paraît essentiel à ce paradis mauricien : c'est l'arbre chalta, ou arbre à pain, appelé par Laure « l'arbre du bien et du mal » (CDO, p. 12) ${ }^{31}$. Les enfants y montent, la nuit surtout, pour écouter la mer, penchés sur l'infini phosphorescent dans l'obscurité qui les interpelle. Il est aussi l'arbre cosmique, le pilier du monde qui maintient l'ordre dans l'univers et assure l'unité entre les humains, limités dans leur pouvoir, et l'infini habité par Dieu et les morts. Il est le socle de l'éternité dans un monde qui s'écroule (CDO, p. 23) ${ }^{32}$. Sachant leur ancien domaine converti en exploitation sucrière, le narrateur a toujours l'espoir de retrouver l'arbre fétiche au bout du jardin : il le retrouve, contrairement à la plupart des arbres, imposant et immuable :

Tout le temps que j'ai été au loin, loin de l'abri de ses feuilles, loin de ses branches, cela n'a été pour lui qu'un instant. L'eau des cyclones est passée, les sécheresses, les incendies, et même les hommes qui ont démoli notre maison, qui ont piétiné les fleurs du jardin et qui ont laissé mourir l'eau du bassin et des canaux. Mais lui est resté l'arbre du bien et du mal qui sait tout, qui voit tout. J'ai cherché les marques que nous avions faites, Laure et moi, avec un couteau, pour inscrire nos noms et notre taille. [...] Le temps a cessé de courir. [...] ici le monde ne connaît pas la faim, ni le malheur. La guerre, cela n'existe pas. L'arbre chalta tient le monde au loin, par la force de ses branches. Notre maison a été détruite, notre père est mort, mais rien n'est désespérant puisque j'ai retrouvé l'arbre chalta. Sous lui je peux dormir. [...] (CDO, p. 357)

S'il est abattu, comme Alexis finit par le supposer, «alors il n'y a plus rien de nous sur cette terre, il n'y a plus un seul point de repère » (CDO, p. 374). Reste alors le large, la liberté en mer.

Reste aussi l'affection des trois femmes les plus proches. La lecture de l'histoire sainte par Mam (CDO, pp. 29-32, 99), dont le souvenir revient aux moments difficiles (CDO, p. 355 : Alexis le rappelle à sa mère, atterrée), devient une passerelle vers l'espoir final de se rejoindre dans un au-delà sidéral (CDO, p. 374). La transcendance - dans la version "naturalisée » par Le Clézio : l'infini de l'océan et le mystère du lointain, son défi éternel - aide le protagoniste à supporter les échecs. Pour Alexis et sa famille, le Boucan est un lieu pleinement investi : lieu de mémoire, maison au bord de la mer habitée par les élues de son cœur, ou encore vaisseau au timon duquel il veille, tel un nouvel Argonaute, dans le sillage de son père $(\mathrm{CDO}, \mathrm{p}$. 374) et du double du père mort, le capitaine Bradmer, resté sans sépulture au large de Rodrigues (CDO, pp. 341-342).

${ }^{31}$ Le narrateur l'appelle aussi « l'arbre de Laure » (CDO, p. 23).

${ }^{32}$ L'importance de l'arbre cosmique a été décrite surtout par M. Eliade, Traité d'histoire des religions, Payot, Paris 1975, passim. 


\section{CONCLUSION}

Appuyé sur l'affection de trois femmes, mortes ou vivantes, proches ou lointaines, et sur l'amitié de deux hommes disparus, fidèles à leur rêve, Alexis, même physiquement épuisé, trouve toujours des forces spirituelles pour à nouveau affronter le destin. Par contre, le narrateur immature des Noces de la vanille vit un cauchemar et non l'ascétisme heureux ${ }^{33}$ ou le dépouillement dans la liberté que cultive le héros leclézien. Hyperréalisme contre utopie, l'un et l'autre des romans sont teints d'onirisme, style poétique par excellence. Ils assument un passage fluide, appuyé sur un fétiche (l'arbre chalta) ou un détail cauchemardesque (le sang et le rouge de la crise chez Masson), le passage du mythe ancien à son actualisation.

Les nouvelles Virginie s'effacent par une fatalité moins transcendante que celle qui frappait leur prototype. Et les Paul modernes ? Ils sont d'une fidélité à toute épreuve : fidélité à Dieu (Raoul) ou à leur amie. Si Alexis trouve la force de repartir à zéro, c'est grâce à ces femmes qu'il a derrière lui et qui lui ont enseigné un ordre immuable, quoique invisible. Si le petit ami de Marie-Thérèse ne sait résister à l'emprise de l'Autre, l'Ennemi (incarné par un intendant mal aimé et malade), ni protéger contre les appétits d'Esparon sa bien-aimée, c'est que «les fiancés de la vanille » ont des parents veules, incapables de leur insuffler de la foi ni de leur servir d'appui. Paul et Virginie, ce qu'on perd de vue aujourd'hui, est l'histoire d'une famille : le mythe du couple repose sur celui d'une demeure bienheureuse ${ }^{34}$, le commencement de tout amour capable de durer, de braver la mort même. Mais pour y parvenir, une condition doit être remplie. Alexis la formule, en prolongeant la voix du vieil ami dans l'hypotexte bernardinien ${ }^{35}:$ «Peut-être que nous braverons l'interdit, et nous irons jusqu'à Saint-Brandon, là où le capitaine Bradmer et son timonier ont trouvé leur refuge ? De l'autre côté du monde, dans un lieu où l'on ne craint plus les signes du ciel, ni la guerre des hommes » (CDO, p. 375). Braver l'interdit jusqu'au bout : rester fidèle à sa voix intérieure.

Mythe fondateur de l'imaginaire collectif de l'océan Indien, l'histoire de Paul et Virginie ne cesse d'alimenter la création littéraire dans ou sur les îles Mascareignes. Les trois romans examinés montrent comment le mythe des adolescents amoureux a évolué en plus d'un siècle. Sa plasticité à porter les tensions et les nostalgies du

${ }^{33}$ D. Barbier, Le Chercheur d'or, collection « Connaissance d'une œuvre », Bréal, Rosny 2005, p. 17.

${ }^{34}$ Comme Clarens de Rousseau, la «petite société » (les six habitants de la vallée) reçoit une lumière utopique. Voir J.-M. Racault, « Introduction » à Paul et Virginie (P\&V, p. 36).

35 Paul, vivant dans une enclave de l'île de France, aurait l'avantage de décider de sa vie en colon libre : «Vous êtes dans un pays [...] où vous pouvez être impunément bon, vrai, sincère, instruit, patient, tempérant, chaste, indulgent, pieux, sans qu'aucun ridicule vienne flétrir votre sagesse, qui n'est encore qu'en fleur ", assure le vieillard ami (P\&V, p. 219). Au vingtième siècle, la société coloniale n'offre plus aucun champ de liberté : Masson et Le Clézio en témoignent. L'idylle est finie, y compris dans l'imaginaire. 
temps lui permet de surnager à une époque où le roman bernardinien, son lieu de naissance, serait tombé dans l'oubli depuis longtemps ${ }^{36}$.

Chez les auteurs implantés dans les îles, la figure des deux enfants s'émousse [...] S'impose l'image d'un anti-éden, comme un contre-cliché qui réfute toute idéalisation utopique d'un monde primitif à l'abri du reste de l'humanité. L'heure est à la recherche d'une représentation du monde insulaire qui en dénonce les dérives,

avance Valérie Magdelaine-Andrianajafitrimo ${ }^{37}$. Au fil des siècles et des guerres, menées en Europe, le lecteur constate l'impuissance grandissante du mythe à pallier la désagrégation affective des humains - en famille, en couple, entre frère et sœur. En même temps, autonomes, les anciennes colonies demandent à régler leurs comptes avec la métropole.

Une fois reconnu, « le rapport de filiation et de rupture ambigu que la littérature indianocéanique entretient avec la France et son canon littéraire »- pour reprendre encore les mots de Valérie Magdelaine-Andrianajafitrimo ${ }^{38}$ — invite à réactualiser, dans un nouveau contexte socio-culturel, à travers un style et un lexique différents, ce que la connaissance des lettres classiques nous a apporté. Ainsi, un pont se crée entre la philologie romane et les études francophones.

\section{SOURCES D'EXEMPLES}

Azéma, G., Noëlla, Librairie de L. Hachette et Cie, Paris 1864. (N)

Bernardin de Saint-Pierre, J.-H., Paul et Virginie, J.-M. Racault (dir.), Union Générale Française, collection « Le Livre de poche classique », Paris 1999. (P\&V)

Bernardin de Saint-Pierre, J.-H., Paul et Virginie, C. Duflo (dir.), [dans :] Euvres complètes, t. 1. Romans et contes, J.-M. Racault et al. (dir.), Classiques Garnier, Paris 2014, pp. 103-434. (1OC)

Le Clézio, J.M.G., Le Chercheur d'or, Éditions Gallimard, collection « Folio », Paris 1985. (CDO) Masson, L., Les Noces de la vanille. Roman, Éditions de l'océan Indien / Robert Laffont, Port-Louis (île Maurice) / Paris 1981. (NV)

${ }^{36}$ Même s'il a bénéficié d'un intérêt croissant de la part de chercheurs, en particulier grâce aux analyses de Jean Fabre, de Jean Ehrard, et de Jean-Michel Racault, organisateur de colloques bernardiniens et responsable des Euvres complètes dont le premier volume, paru chez Classiques Garnier, comprend Paul et Virginie (édité par Colas Duflo).

${ }^{37}$ Cela en rapport avec le roman de J.-F. Samlong (V. Magdelaine-Andrianajafitrimo, op. cit., p. 485)

38 Ibidem. 


\section{THE MYTH OF PAUL AND VIRGINIE IN THREE FRANCOPHONE NOVELS OF THE INDIAN OCEAN}

\section{Summary}

Ex oriente lux? From the Southern Tropics in any case, since certain myths from former times, forgotten and buried under indifference, come back to us rejuvenated and transformed. In this article, we treat one myth - 'myth' given the extent of its cultural hypertext - which arose, strangely but almost necessarily, in an ancient French colony: the Île-de-France (Mauritius). It may seem fairly obvious that Paul and Virginie (hero and heroine of Bernardin de Saint-Pierre's eponymous novel) should have returned to haunt the literature of the Île-de-France and her "sister island", La Réunion. We examine three novels: the first transcribes the idyllic couple in terms of a realism based on a form of local colour (Georges Azéma, Noëlla, 1874). The second ends up destroying the pastoral eclogue of Bernardin de Saint-Pierre (Loys Masson, Les Noces de la vanille, 1962, English title: The Overseer). The third novel, Le Chercheur d'or by J.M.G. Le Clézio (1985, English title: The Collector), abandons the island setting in order to preserve the myth. Whether colonial or postcolonial, the old myth, dressed in new clothes, invites us to a dialogue between different centuries and different cultures.

Key words: Loys Masson, Le Clézio, Georges Azéma, literary myth, Creole, Eden, colonial. 